\title{
Factors Associated with the Immunogenicity of Anti-Tumor Necrosis Factor Agents in Pediatric Patients with Inflammatory Bowel Disease
}

\author{
Ju Young Kim ${ }^{1,2}$, Yoon Lee ${ }^{3}$, Byung-Ho Choe ${ }^{1,4}$, and Ben Kang ${ }^{1,4}$ \\ ${ }^{1}$ Department of Pediatrics, School of Medicine, Kyungpook National University, Daegu, ${ }^{2}$ Department of Pediatrics, Eulji University \\ School of Medicine, Daejeon, ${ }^{3}$ Department of Pediatrics, Korea University School of Medicine, Seoul, and ${ }^{4}$ Crohn's and Colitis \\ Association in Daegu-Gyeongbuk (CCAiD), Daegu, Korea
}

\section{Article Info}

Received April 22, 2020

Revised August 23, 2020

Accepted August 24, 2020

Published online October 8, 2020

\section{Corresponding Author}

Ben Kang

ORCID https://orcid.org/0000-0002-8516-9803

E-mail benkang@knu.ac.kr

Ju Young Kim and Yoon Lee contributed equally to this work as first authors.
Background/Aims: Anti-drug antibodies (ADAs) can develop during treatment with anti-tumor necrosis factor (TNF) agents. We aimed to investigate the factors associated with immunogenicity of anti-TNF agents in pediatric patients with inflammatory bowel disease (IBD) and observe the clinical course of ADA-positive patients.

Methods: Pediatric IBD patients receiving maintenance treatment with anti-TNF agents who had been tested for ADAs against infliximab (IFX) or adalimumab (ADL) were included in this crosssectional study. Factors associated with ADA positivity were investigated by analyzing clinicodemographic, laboratory, and treatment-related factors.

Results: A total of 76 patients (Crohn's disease, 65; ulcerative colitis, 11) were included. Among these, 59 and 17 patients were receiving IFX and ADL, respectively. ADAs were found in 10 patients (13.2\%), all of whom were receiving IFX. According to multivariable logistic regression analysis, the IFX trough level (TL) was associated with ADA positivity (odds ratio, $0.25 ; 95 \%$ confidence interval $[\mathrm{Cl}], 0.08$ to $0.51 ; p=0.002$ ). According to the receiver operating characteristic analysis, the optimal cutoff of the IFX TLs for stratifying patients based on the presence of ADAs against IFX was $1.88 \mu \mathrm{g} / \mathrm{mL}$ (area under curve, $0.941 ; 95 \% \mathrm{Cl}, 0.873$ to 1.000 ; sensitivity, $80.0 \%$; specificity, $95.9 \%$; $\mathrm{p} 00.001)$. Among the 10 patients with ADAs against IFX, five patients $(50 \%)$ switched to ADL within 1 year, while five patients $(50 \%)$ kept receiving IFX. Transient ADAs were observed in three patients (30\%).

Conclusions: IFX TL was the only factor associated with ADA formation in pediatric IBD patients receiving IFX. Future studies based on serial and proactive therapeutic drug monitoring are required in the future. (Gut Liver 2021;15:588-598)

Key Words: Infliximab; Adalimumab; Crohn disease; Ulcerative colitis; Children

\section{INTRODUCTION}

Inflammatory bowel disease (IBD), including Crohn's disease (CD) and ulcerative colitis, is a chronic disease of multifactorial etiology characterized by repetitive episodes of relapse and remission of gastrointestinal symptoms. ${ }^{1}$ Approximately $25 \%$ of patients with IBD develop IBDcompatible symptoms before reaching the age of 20 years, and IBD in patients $<20$ years old typically exhibit a more aggressive phenotype than that seen in adults. ${ }^{2-4}$ Early effective treatment is therefore crucial in pediatric IBD patients. ${ }^{5,6}$ Likewise, treatment strategies in pediatric IBD have evolved to introduce anti-tumor necrosis factor (TNF) agents earlier in the disease course.

However, primary nonresponse and secondary loss of response (LOR) is another problem that physicians face during treatment with these agents, with less than $40 \%$ of patients capable of sustaining clinical remission at 1 year. ${ }^{8}$ This may in part be explained by the occasional development of immunogenicity to anti-TNF agents. The develop- 
ment of anti-drug antibodies (ADAs) is reported to occur in up to $65.3 \%$ of patients receiving infliximab (IFX), and in up to $38.0 \%$ of those receiving adalimumab (ADL). ${ }^{9}$ The formation of ADAs, which possess neutralizing or binding properties, not only interferes with the binding of agents to TNF- $\alpha$ but also accelerates drug clearance by the reticuloendothelial system. ${ }^{8}$ Moreover, recent evidence suggests that the development of ADAs could be a result of insufficient IFX exposure. ${ }^{10,11}$ Even so, an association between the presence of ADAs and poor clinical outcomes has been reported, and recent data from a meta-analysis shows that patients with ADAs against IFX are three times more likely to lose response compared with patients who have not developed ADAs. $^{12}$

Apart from the association with drug concentration, certain patient factors, such as the concomitant administration of immunomodulators during anti-TNF treatment, have also shown an association with ADA formation in adults; ${ }^{8,13-15}$ however, available data are limited for pediatric IBD patients. Therefore, we aimed to investigate factors associated with the development of immunogenicity to antiTNF agents in pediatric patients with IBD and the clinical course of patients with ADAs.

\section{MATERIALS AND METHODS}

\section{Patients and study design}

This study was approved by the Institutional Review Board of Kyungpook National University Chilgok Hospital (IRB number: 2017-09-026), and was performed in accordance with the Declaration of Helsinki.

This was a retrospective cross-sectional study performed at the Department of Pediatrics, Kyungpook National University Chilgok Hospital from March to October 2017. Subjects included were pediatric IBD patients who had been diagnosed with IBD before reaching the age of 18 , who were receiving maintenance treatment with antiTNF agents, namely IFX or ADL, and for whom test results were available on ADAs as well as data on anti-TNF agent trough levels (TLs). Patients who had started antiTNF therapy only after reaching the age of 18, and those who had not been tested for ADAs were excluded. CD and ulcerative colitis were diagnosed according to the revised Porto Criteria of the European Society for Paediatric Gastroenterology, Hepatology, and Nutrition. ${ }^{16}$

Clinicodemographic and treatment-related data including sex, diagnosis age, diagnosis, disease duration from diagnosis to anti-TNF initiation, duration of anti-TNF treatment, anti-TNF type, previous anti-TNF treatment, concomitant medication, and dose intensification dur- ing maintenance were collected from electronic medical charts. Clinical scores including Pediatric Crohn's Disease Activity Index and Pediatric Ulcerative Colitis Activity Index scores, routine laboratory test results including complete blood cell count, erythrocyte sedimentation rate, Creactive protein, chemistry profiles, and fecal calprotectin results were collated together with TL and ADA results. Serum samples for TL and ADA testing had been obtained during maintenance treatment with anti-TNF agents at trough point before the scheduled infusion in addition to samples for routine laboratory tests. Samples had been obtained not serially but just once for all patients, while for those with positive ADAs at initial tests additional samples were obtained 1 year afterwards. Serum TLs and ADAs were measured using enzyme-linked immunosorbent assay kits from Matriks Biotek Laboratories (Ankara, Turkey) and Immundiagnostik AG (Bensheim, Germany), respectively. ${ }^{17,18}$

An ADA level $>10 \mathrm{AU} / \mathrm{mL}$ was defined as ADA positive, while an ADA level $\leq 10 \mathrm{AU} / \mathrm{mL}$ was defined as a negative ADA, for both IFX and ADL. Subtherapeutic TLs for IFX and ADL were defined as $<5$ and $<7.5 \mu \mathrm{g} / \mathrm{mL}$, respectively. ${ }^{19}$ Clinical remission was defined as a Pediatric Crohn's Disease Activity Index $<10$ for CD and Pediatric Ulcerative Colitis Activity Index $<10$ for ulcerative colitis. Patients were divided into two groups according to ADA positivity, and investigation of factors associated with ADA positivity was carried out. In patients positive for ADAs, the treatment and clinical course were further observed over the following 12 months.

\section{Statistical analysis}

The Student t-test and Wilcoxon rank-sum test were used for the comparison of continuous variables between groups, and the chi-square test and Fisher exact test were used for the comparison of categorical variables between groups. Data are expressed as mean \pm standard deviation for factors exhibiting a normal distribution, and median (interquartile range) for factors exhibiting a non-normal distribution. Logistic regression analyses were performed to investigate factors associated with ADA positivity. Univariate logistic regression analysis was first carried out to investigate the crude odds ratio (OR) for each factor, and multivariate logistic regression with a stepwise selection procedure was performed to investigate adjusted ORs for significant risk factors after adjusting for possible confounders. As the stepwise procedure was applied, only factors showing a univariate-level significance of $\mathrm{p}<0.1$ were included in the multivariate logistic model. Receiver operating characteristic curve analysis was performed to determine the optimal cutoff of factors that could best 
stratify patients with and without ADAs. Probability p-values $<0.05$ were considered to imply statistical significance. Statistical analysis was carried out using the R software (version 3.2.3; R Foundation. Vienna, Austria).

\section{RESULTS}

\section{Baseline characteristics}

A total of 92 patients were receiving maintenance treatment with anti-TNF agents during the study period. Among them 76 patients (82.6\%) who met the inclusion criteria were included in this retrospective cross-sectional study. Among the 76 patients, 49 (64.5\%) were males, and $65(85.5 \%)$ were diagnosed with CD. Fifty-nine patients (77.6\%) were receiving IFX, and $56(73.7 \%)$ were receiving combination treatment with immunomodulators. Additional baseline characteristics are shown in Table 1.

\section{Comparison of factors according to ADA positivity}

ADAs were detected in 10 patients (13.2\%). All patients with ADAs were receiving IFX for maintenance treat- ment. Comparison of factors between patients according to ADA positivity revealed that the rate of clinical remission was significantly lower in patients who were ADA-

Table 1. Baseline Characteristics

\begin{tabular}{lc}
\hline \multicolumn{1}{c}{ Variable } & Value \\
\hline Diagnosis & \\
CD & $65(85.5)$ \\
UC & $11(14.5)$ \\
Male sex & $49(64.5)$ \\
Age at diagnosis, yr & $14.1(12.1-15.5)$ \\
Age at study inclusion, yr & $17.0(15.4-18.7)$ \\
Duration from diagnosis to anti-TNF initiation, yr & $0.1(0.0-0.5)$ \\
Duration of anti-TNF treatment, yr & $2.5(1.4-3.6)$ \\
Anti-TNF agent & \\
Adalimumab & $17(22.4)$ \\
Infliximab & $59(77.6)$ \\
Previous treatment with other anti-TNF agent & $2(2.6)$ \\
Dose intensification & $20(26.3)$ \\
Concomitant immunomodulators & $56(73.7)$ \\
Concomitant corticosteroids & 0 \\
\hline
\end{tabular}

Data are presented as number (\%) or median (interquartile range). CD, Crohn's disease; UC, ulcerative colitis; TNF, tumor necrosis factor.

Table 2. Comparison of Factors between Patients Stratified According to the Presence of an Immune Response to Anti-TNF Agents

\begin{tabular}{|c|c|c|c|}
\hline Variable & ADA-negative $(n=66)$ & ADA-positive $(n=10)$ & p-value \\
\hline Male sex & $41(62.1)$ & $8(80.0)$ & 0.455 \\
\hline Diagnosis & & & 0.310 \\
\hline $\mathrm{CD}$ & $58(87.9)$ & $7(70.0)$ & \\
\hline UC & $8(12.1)$ & $3(30.0)$ & \\
\hline Age at diagnosis, yr & $14.4(12.2-15.6)$ & $13.3(9.9-14.9)$ & 0.318 \\
\hline Age at study inclusion, yr & $17.0(15.4-18.8)$ & $17.0(14.4-18.7)$ & 0.695 \\
\hline Duration from diagnosis to anti-TNF treatment initiation, yr & $0.1(0.0-0.5)$ & $0.0(0.0-0.5)$ & 0.605 \\
\hline Duration of anti-TNF treatment, yr & $2.5(1.3-3.4)$ & $3.0(1.8-4.1)$ & 0.398 \\
\hline Anti-TNF agent & & & 0.157 \\
\hline Adalimumab & $17(22.4)$ & 0 & \\
\hline Infliximab & $49(74.2)$ & $10(100.0)$ & \\
\hline Previous treatment with other anti-TNF agent & $2(3.0)$ & 0 & 1.000 \\
\hline Dose intensification & $16(24.2)$ & $4(40.0)$ & 0.440 \\
\hline Concomitant immunomodulator & $49(74.2)$ & $7(70.0)$ & 1.000 \\
\hline Clinical remission & $62(93.9)$ & $6(60.0)$ & 0.007 \\
\hline $\mathrm{WBC}, / \mu \mathrm{L}$ & $6,260(5,250-7,220)$ & $8,185(7,460-8,360)$ & 0.004 \\
\hline Hematocrit, \% & $40.5 \pm 3.7$ & $41.6 \pm 4.0$ & 0.387 \\
\hline Platelet count, $\times 10^{3} / \mu \mathrm{L}$ & $284.7 \pm 60.2$ & $314.6 \pm 84.6$ & 0.171 \\
\hline Albumin, g/dL & $4.4(4.2-4.5)$ & $4.2(4.1-4.4)$ & 0.116 \\
\hline $\mathrm{CRP}, \mathrm{mg} / \mathrm{dL}$ & $0.0(0.0-0.1)$ & $0.2(0.1-0.5)$ & 0.017 \\
\hline $\mathrm{ESR}, \mathrm{mm} / \mathrm{hr}$ & $7(3-16)$ & $11.5(3-18)$ & 0.578 \\
\hline $\mathrm{FC}, \mathrm{mg} / \mathrm{kg}$ & $55.2(21.2-208.4)$ & $54.0(16.3-808.5)$ & 0.641 \\
\hline Infliximab TL, $\mu \mathrm{g} / \mathrm{mL}$ & $5.3(3.3-7.5)$ & $1.0(0.0-1.7)$ & $<0.001$ \\
\hline Adalimumab $T L, \mu \mathrm{g} / \mathrm{mL}$ & $8.3(6.6-11.2)$ & NA & NA \\
\hline Subtherapeutic TL & $31(47.0)$ & $10(100.0)$ & 0.001 \\
\hline Infliximab ADA level, AU/mL & $3.3(2.7-4.3)$ & $30.6(17.3-86.3)$ & $<0.001$ \\
\hline Adalimumab ADA level, AU/mL & $1.5(1.3-1.5)$ & NA & NA \\
\hline
\end{tabular}

Data are presented as number (\%), median (interquartile range), or mean \pm SD.

TNF, tumor necrosis factor; ADA, anti-drug antibody; CD, Crohn's disease; UC, ulcerative colitis; WBC, white blood cell; CRP, C-reactive protein; ESR, erythrocyte sedimentation rate; FC, fecal calprotectin; TL, trough level; NA, not applicable. 
positive compared with those who were ADA-negative (60.0\% vs $93.9 \%$; $p=0.007$ ). Significant differences between the two groups were also observed with regard to white blood cell (WBC) counts (median $8,185 / \mu \mathrm{L}$ vs $6,260 / \mu \mathrm{L}$; $\mathrm{p}=0.004$ ), C-reactive protein (median $0.2 \mathrm{mg} / \mathrm{dL}$ vs $0.0 \mathrm{mg} /$ $\mathrm{dL} ; \mathrm{p}=0.017$ ), IFX TLs (median $1.0 \mu \mathrm{g} / \mathrm{mL}$ vs $5.3 \mu \mathrm{g} / \mathrm{mL}$; $\mathrm{p}<0.001$ ), and ADA levels against IFX (median $3.3 \mathrm{AU} / \mathrm{mL}$ vs $30.6 \mathrm{AU} / \mathrm{mL} ; \mathrm{p}<0.001$ ) (Table 2). However, no significant difference was observed with regard to the proportion of patients receiving immunomodulators between patients with and without $\operatorname{ADAs}(74.2 \%$ vs $70.0 \%$; $\mathrm{p}=1.000$ ).

An additional comparison was performed of patients divided according to anti-TNF type. Among the baseline characteristics, the duration of anti-TNF treatment was significantly longer in patients who were receiving IFX compared with those receiving ADL (3.0 \pm 1.3 years vs

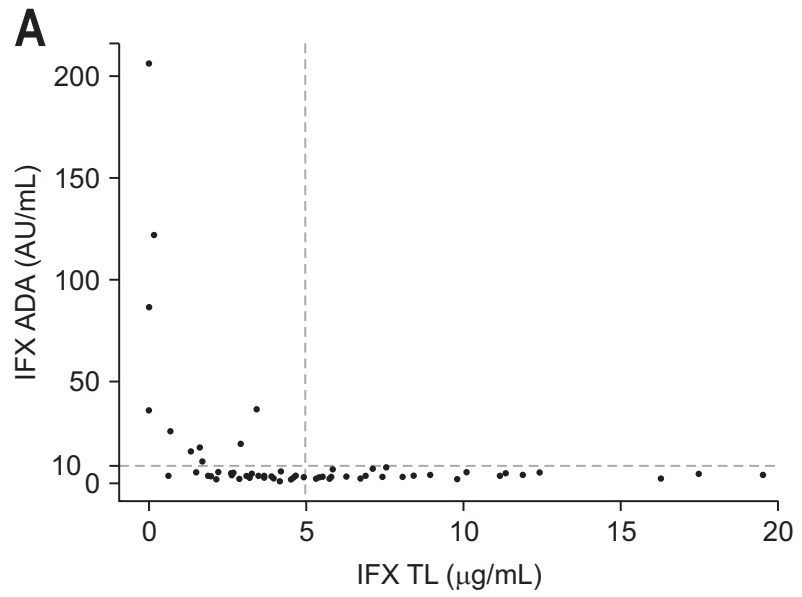

$0.9 \pm 0.5$ years; $\mathrm{p}<0.001)$. Significant differences between patients receiving IFX and ADL were also observed with regard to erythrocyte sedimentation rate levels (median [interquartile range] IFX vs ADL: $9.0 \mathrm{~mm} / \mathrm{hr}$ [4.0 to 16.5] vs $4.0 \mathrm{~mm} / \mathrm{hr}$ [2.0 to 7.0 ]; $\mathrm{p}=0.011$ ). The ADA levels for patients receiving ADL were all $<10 \mathrm{AU} / \mathrm{mL}$, and ADL TLs were $\geq 5 \mu \mathrm{g} / \mathrm{mL}$ (Fig. 1).

\section{Factors associated with ADA positivity}

According to univariate logistic regression analysis, WBC count was the only significant factor associated with ADA positivity (OR, 1.63; 95\% confidence interval [CI], 1.11 to $2.50 ; \mathrm{p}=0.015)$. Meanwhile, $\mathrm{C}$-reactive protein and fecal calprotectin (FC) both showed p-values $<0.1$ and were included in the multivariate analysis along with WBC count (Table 3). According to multivariate logistic regres-

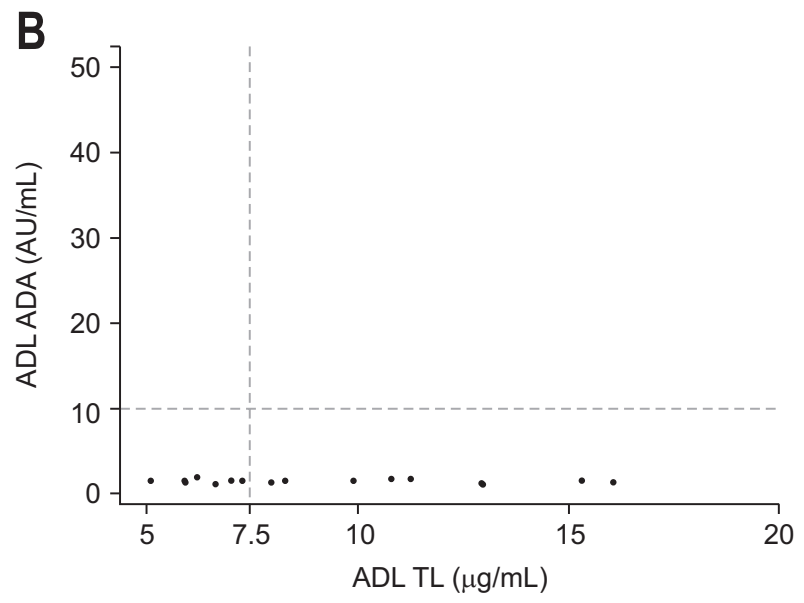

Fig. 1. Scatter diagram of TLs and ADAs among patients receiving (A) IFX and (B) ADL.

$T L$, trough level; ADA, anti-drug antibody; IFX, infliximab; ADL, adalimumab.

Table 3. Factors Associated with Immunogenicity of Anti-TNF Agents

\begin{tabular}{|c|c|c|c|c|c|c|}
\hline \multirow{2}{*}{ Variable } & \multicolumn{3}{|c|}{ Univariate analysis } & \multicolumn{3}{|c|}{ Multivariate analysis with stepwise selection } \\
\hline & $\mathrm{OR}$ & $95 \% \mathrm{Cl}$ & p-value & $\mathrm{OR}$ & $95 \% \mathrm{Cl}$ & p-value \\
\hline Sex (male) & 2.44 & $0.56-17.03$ & 0.283 & & & \\
\hline Diagnosis (UC) & 3.11 & $0.58-13.97$ & 0.149 & & & \\
\hline Age at diagnosis & 0.88 & $0.73-1.09$ & 0.220 & & & \\
\hline Age at study inclusion & 0.92 & $0.76-1.13$ & 0.378 & & & \\
\hline Duration from diagnosis to anti-TNF treatment initiation & 1.02 & $0.46-1.64$ & 0.943 & & & \\
\hline Duration of anti-TNF treatment & 1.17 & $0.74-1.82$ & 0.494 & & & \\
\hline Dose intensification (yes) & 2.08 & $0.48-8.25$ & 0.299 & & & \\
\hline Concomitant immunomodulator (yes) & 0.81 & $0.20-4.08$ & 0.777 & & & \\
\hline WBC count & 1.63 & $1.11-2.50$ & 0.015 & 1.98 & $1.26-3.39$ & 0.006 \\
\hline Hematocrit & 1.09 & $0.91-1.33$ & 0.384 & & & \\
\hline Platelet count & 1.01 & $1.00-1.02$ & 0.176 & & & \\
\hline Albumin & 0.08 & $0.00-2.40$ & 0.166 & & & \\
\hline CRP & 5.58 & $0.65-44.23$ & 0.094 & & & \\
\hline ESR & 1.00 & $0.93-1.05$ & 0.998 & & & \\
\hline $\mathrm{FC}$ & 1.11 & $0.97-1.25$ & 0.097 & 1.14 & $1.00-1.31$ & 0.044 \\
\hline
\end{tabular}

TNF, tumor necrosis factor; OR, odds ratio; Cl, confidence interval; UC, ulcerative colitis; WBC, white blood cell; CRP, C-reactive protein; ESR, erythrocyte sedimentation rate; FC, fecal calprotectin. 
sion analysis with stepwise selection, WBC count and FC were significantly associated with ADA positivity (OR, 1.98; $95 \% \mathrm{CI}, 1.26$ to $3.39 ; \mathrm{p}=0.006$ and $\mathrm{OR}, 1.14 ; 95 \% \mathrm{CI}$, 1.00 to $1.31 ; \mathrm{p}=0.044$, respectively) (Table 3 ).

Because ADAs were observed only in patients receiving IFX, a sub-analysis was additionally performed. According to univariate analysis, WBC count and IFX TL were significantly associated with ADA positivity (OR, 1.51; 95\% CI, 1.04 to $2.29 ; \mathrm{p}=0.039$ and $\mathrm{OR}, 0.25 ; 95 \% \mathrm{CI}, 0.08$ to 0.51 ; $\mathrm{p}=0.002$, respectively). These two factors along with platelet count and FC, which both showed a p-value $<0.1$, were included in the multivariate analysis (Table 4). According to multivariate logistic regression analysis with stepwise selection, IFX TL was the only factor associated with the ADA positivity (OR, 0.13 ; $95 \% \mathrm{CI}, 0.01$ to $0.42 ; \mathrm{p}=0.015$ ) (Table 4).

A further analysis was carried out to reveal the optimal cutoff of factors that could best stratify patients with and without ADAs in patients receiving IFX. According to receiver operating characteristic curve analysis, the optimal cutoff level indicating ADA positivity was $1.88 \mu \mathrm{g} / \mathrm{mL}$ for IFX TL (area under curve, $0.941 ; 95 \%$ CI, 0.873 to 1.000 ; sensitivity, $80.0 \%$; specificity, $95.9 \%$; positive predictive value, $80.0 \%$; negative predictive value, $95.9 \%$; $\mathrm{p}<0.001$ ) (Fig. 2).

\section{Treatment and clinical course in patients with ADAs}

Among the 10 patients who had developed ADAs against IFX, secondary LOR was observed in four patients (Fig. 3). IFX doses and intervals were $5 \mathrm{mg} / \mathrm{kg}$ every 4 weeks for three of these four patients, and the remaining patient was receiving IFX by regular doses and intervals of $5 \mathrm{mg} / \mathrm{kg}$ every 8 weeks and also experienced an anaphylactic infusion reaction. IFX was switched to ADL in these four patients, while IFX was continued in the other six patients who were in clinical remission. In these six patients, secondary LOR occurred in two patients within a year, and so, one patient was switched to ADL, while symptoms improved in the other after dose intensification to $10 \mathrm{mg} /$ $\operatorname{kg}$ every 8 weeks. The other four patients sustained clinical remission for a year without secondary LOR.

Among the five patients who kept receiving IFX, TLs, and ADAs were measured 1 year after the initial measure-

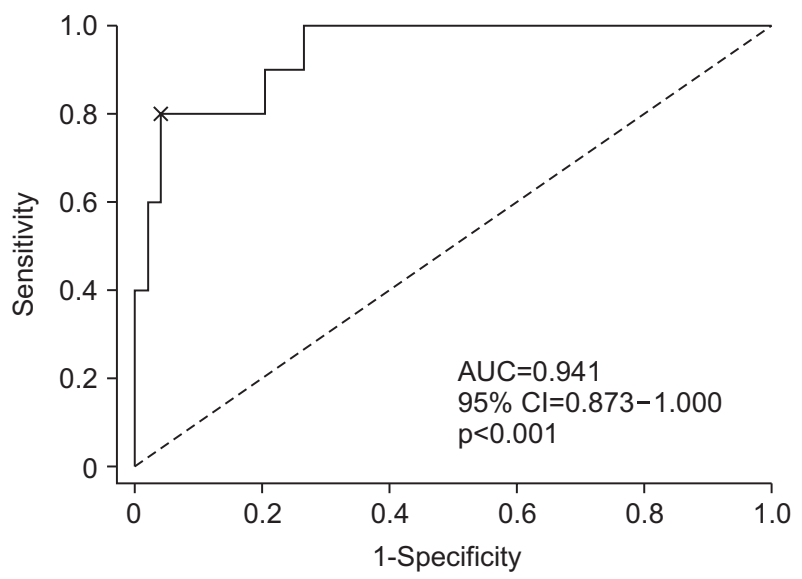

Fig. 2. Receiver operating characteristic curve of infliximab trough levels stratifying patients with and without anti-drug antibodies against infliximab.

$\mathrm{AUC}$, area under curve; $\mathrm{Cl}$, confidence interval.

Table 4. Factors Associated with Immunogenicity of Infliximab ( $n=59$ )

\begin{tabular}{|c|c|c|c|c|c|c|}
\hline \multirow{2}{*}{ Variable } & \multicolumn{3}{|c|}{ Univariate analysis } & \multicolumn{3}{|c|}{ Multivariate analysis with stepwise selection } \\
\hline & $\mathrm{OR}$ & $95 \% \mathrm{Cl}$ & p-value & OR & $95 \% \mathrm{Cl}$ & p-value \\
\hline Sex (male) & 3.00 & $0.67-21.27$ & 0.192 & & & \\
\hline Diagnosis (UC) & 2.57 & $0.47-12.00$ & 0.234 & & & \\
\hline Age at diagnosis & 0.89 & $0.71-1.11$ & 0.278 & & & \\
\hline Age at study inclusion & 0.88 & $0.7-1.09$ & 0.211 & & & \\
\hline Duration from diagnosis to anti-TNF treatment initiation & 1.04 & $0.45-1.79$ & 0.211 & & & \\
\hline Duration of anti-TNF treatment & 0.88 & $0.51-1.47$ & 0.640 & & & \\
\hline Dose intensification (yes) & 2.30 & $0.51-9.62$ & 0.254 & & & \\
\hline Concomitant immunomodulator (yes) & 1.13 & $0.27-5.78$ & 0.870 & & & \\
\hline WBC count & 1.51 & $1.04-2.29$ & 0.039 & & & \\
\hline Hematocrit & 1.16 & $0.94-1.47$ & 0.205 & & & \\
\hline Platelet count & 1.01 & $1.00-1.02$ & 0.084 & 1.03 & $1.00-1.07$ & 0.089 \\
\hline Albumin & 0.07 & $0.00-2.18$ & 0.159 & & & \\
\hline CRP & 4.33 & $0.49-35.53$ & 0.158 & & & \\
\hline ESR & 0.99 & $0.92-1.05$ & 0.731 & & & \\
\hline FC & 1.13 & $0.98-1.33$ & 0.084 & & & \\
\hline Infliximab TL & 0.25 & $0.08-0.51$ & 0.002 & 0.13 & $0.01-0.42$ & 0.015 \\
\hline
\end{tabular}

$\mathrm{OR}$, odds ratio; $\mathrm{Cl}$, confidence interval; UC, ulcerative colitis; TNF, tumor necrosis factor; WBC, white blood cell; CRP, C-reactive protein; ESR, erythrocyte sedimentation rate; FC, fecal calprotectin; TL, trough level. 


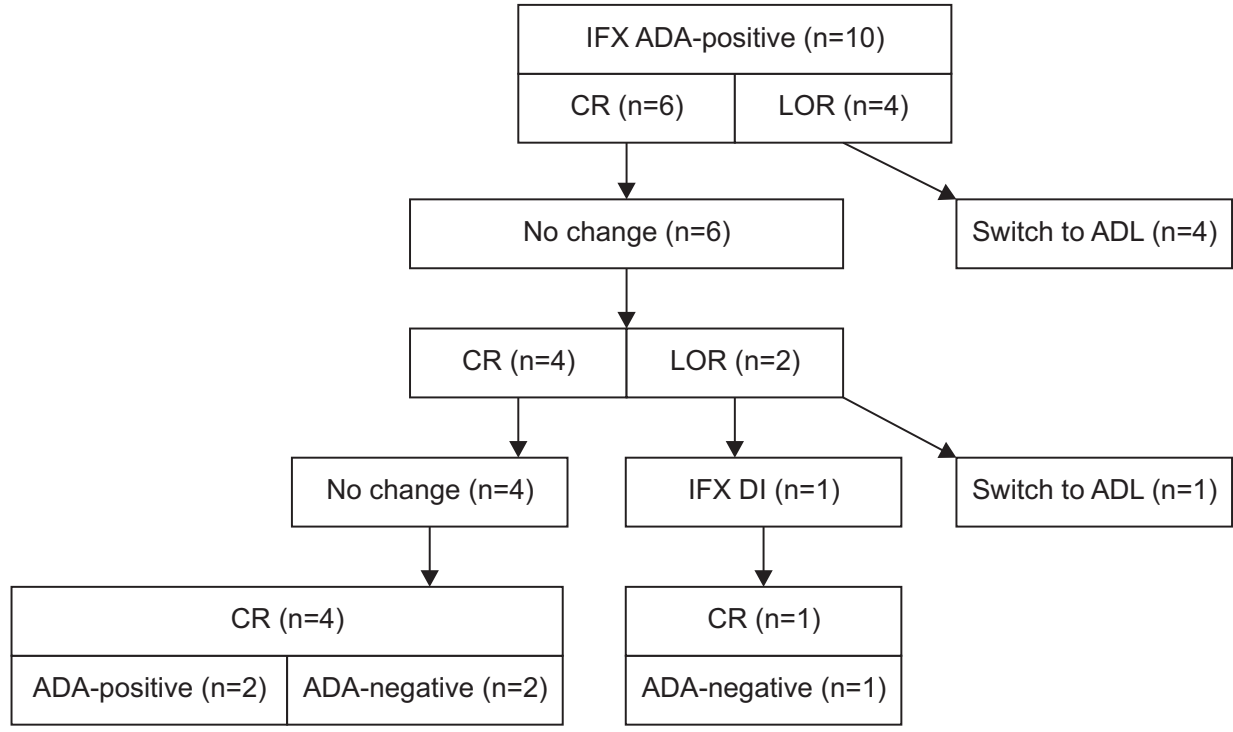

Fig. 3. Flow diagram of the treatment and clinical course of patients with ADAs against IFX.

$A D A$, anti-drug antibody; $C R$, clinical remission; LOR, loss of response; $A D L$, adalimumab; IFX, infliximab; DI, dose intensification. ments. Three patients were negative for ADAs, while ADAs were still detected in two patients (Table 5). Initial ADA titers were lower in the three patients who became ADAnegative at 1 year compared with the seven patients who had experienced secondary LOR and/or who remained ADA-positive at 1 year, revealing a borderline statistical significance $(14.97 \pm 4.37 \mathrm{AU} / \mathrm{mL}$ vs $75.60 \pm 68.82 \mathrm{AU} / \mathrm{mL}$; $\mathrm{p}=0.059$ ).

\section{DISCUSSION}

In this study, we investigated clinicodemographic, laboratory and treatment-related factors potentially associated with immunogenicity to anti-TNF agents in pediatric patients with IBD who had been receiving maintenance treatment with either IFX or ADL. We found a significant association between IFX TLs and ADA positivity in patients receiving IFX, and derived an optimal IFX TL cutoff of $1.88 \mu \mathrm{g} / \mathrm{mL}$ that could best stratify patients with and without ADAs. These results are the first from real-life clinical practice in Korean children with IBD.

Immunogenicity to anti-TNF agents arises when the immune system of the host recognizes the drug as a foreign antigen and develops specific ADAs against it. ${ }^{15}$ In adults, the development of ADAs to IFX occurs in up to $65.3 \%$ in patients with IBD, ${ }^{9}$ while ADAs to IFX have been reported in $8 \%$ to $43 \%$ of pediatric patients with $\mathrm{IBD}^{20}{ }^{20}$ The results of our study are in agreement with these observations, as ADAs were detected in $13.2 \%$ of patients receiving IFX. Meanwhile, no ADAs were detected in patients receiving ADL in this study. Because ADL is a humanized monoclonal antibody, theoretically it should be less immunogenic compared with IFX. However, data from studies from adult patients with IBD show that the risk of immunogenicity to ADL is comparable with that of IFX, which is reported to be up to $38.0 \%{ }^{9}$

There is limited data regarding the immunogenicity to ADL from studies on children with IBD. ADAs to ADL have been reported to occur in $3.3 \%$ to $10.3 \%$ of pediatric patients, ${ }^{21,22}$ which is a relatively lower proportion than that observed among their counterparts receiving IFX and also among adult patients with IBD. The absence of ADAs against ADL observed in our study could be due to the significantly shorter duration of anti-TNF treatment in patients treated with ADL compared with those receiving IFX $(0.9 \pm 0.5$ years vs $3.0 \pm 1.3$ years; $p<0.001)$. However, a recent prospective study of adult patients with $\mathrm{CD}$ receiving $\mathrm{ADL}$ revealed that the development of ADAs against ADL occurred in $79 \%$ of patients by week 14 of ADL initiation. ${ }^{23}$ Further studies in children with IBD are required to elucidate whether the risk and timing of the development of ADAs to ADL differ from those of adults.

The development of ADAs to anti-TNF agents can neutralize the drug by direct binding of neutralizing antibodies or accelerating the clearance of the drug by the reticuloendothelial system via an indirect mechanism of binding of non-neutralizing antibodies to the drug. ${ }^{9,24}$ This causes a decrease of anti-TNF drug levels, which may lead to LOR during treatment. ${ }^{12,25,26}$ Studies in children with IBD have also revealed an association between ADAs and LOR. ${ }^{27,28}$ This was also observed in our study, which revealed significantly lower IFX TLs and significantly lower rates of clinical remission in patients with ADAs against IFX compared with those who were ADA-negative. Furthermore, WBC count and FC were significantly associated with ADA posi- 


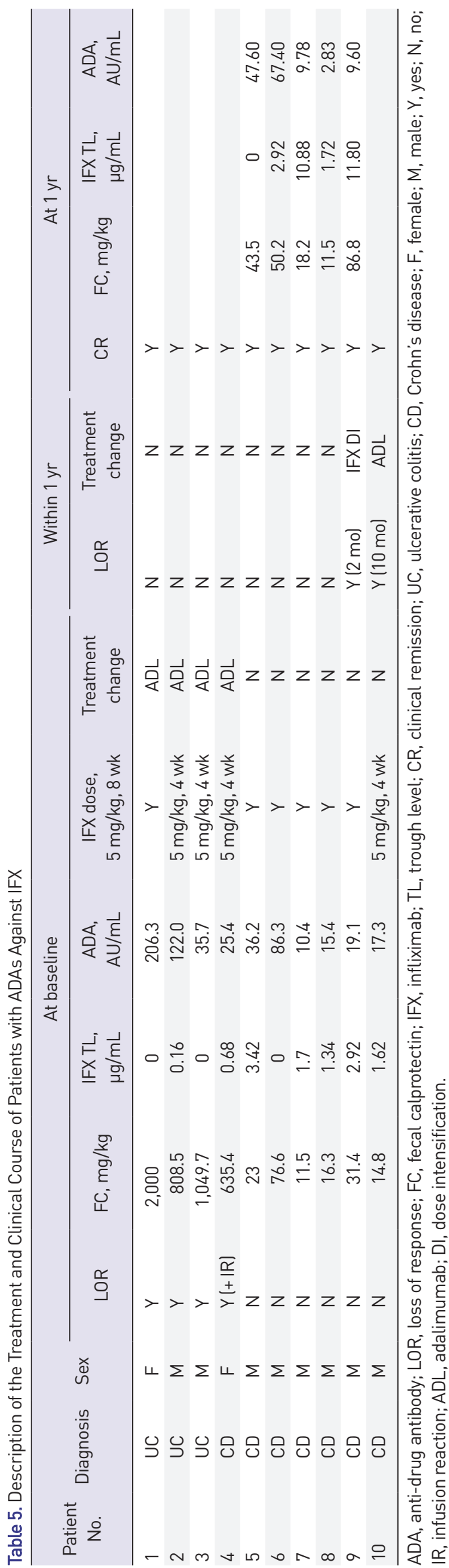

tivity in our study, indicating the negative impact of the presence of ADAs on controlling systemic inflammation as well as mucosal healing. ADAs are also known to be associated with the occurrence of infusion reactions, and may impose a 6-fold higher risk of serious acute infusion reactions as observed in a patient in our study. ${ }^{29}$

Combination therapy with an immunomodulator during treatment with anti-TNF agents is a well-known factor capable of decreasing the risk of ADA formation, leading to higher anti-TNF drug levels and better treatment outcomes in both children and adults with IBD..$^{12,13,30-33}$ In a retrospective multicenter observational study of 229 children with CD receiving IFX, ADA formation was higher in patients on IFX monotherapy compared to those who were on continuous combined immunosuppression $(\mathrm{p}=0.003)$ and had received early combined immunosuppression $(\mathrm{p}=0.008)$ according to Kaplan-Meier analysis (log-rank overall 0.002). ${ }^{31}$ In this study, ADAs to IFX developed in each $42 \%(8 / 19), 10 \%(6 / 62)$, and $14 \%(11 / 81)$ who received IFX monotherapy, on continuous combined immunosuppression, and had received early combined immunosuppression, respectively. In another cross-sectional study of 223 pediatric and young adult patients with IBD on IFX, patients who were currently on combination therapy had a lower rate of detectable ADA to IFX (9.5\%) compared with those on monotherapy (20.0\%) in multivariate analysis (OR, 0.3 ; $95 \%$ CI, 0.1 to 0.7 ; $\mathrm{p}<0.01$ ). ${ }^{32}$ However, we were unable to observe this impact of immunomodulators on the development of ADAs to IFX in our study. This may be due to the small number of patients included, as well as the limitations of the cross-sectional study design. Another possible reason may be due to the fact that all of the patients had started IFX with an immunomodulator although 20 patients had stopped them sometime during maintenance treatment with IFX. Meanwhile, a recent study of children with $C D$ revealed that there was no benefit of adding an immunomodulator to ADL treatment compared with ADL monotherapy. ${ }^{22}$ Further large-scale and longitudinal studies on the impact of concomitant treatment with an immunomodulator during treatment with ADL in children are required for better insight.

Therapeutic drug monitoring (TDM) of anti-TNF agents has emerged as a strategy to optimize and personalize treatment based on measurement of TLs and ADAs. ${ }^{34}$ Reactive TDM, which suggests treatment based on TDM results taken at the point of secondary LOR, is currently recommended in clinical practice. ${ }^{35}$ According to acknowledged treatment algorithms for reactive TDM, when TLs are subtherapeutic and ADAs detectable, optimizing the index therapy is recommended in case of low-level ADAs, and switching to a different drug class plus addition of 
an immunomodulator is recommended in patients with high-level ADAs. ${ }^{19}$ In our study, all four patients in whom secondary LOR had occurred switched to ADL. These decisions had been made clinically, since the actual measurement of TLs and ADAs were conducted afterwards. When retrospectively investigated, decisions based on clinical symptoms were fortunately in line with current guidelines, although the enzyme-linked immunosorbent assay kits we had used for measuring ADAs did not provide a cutoff for discriminating between high- and low-level ADAs.

Transient ADAs can spontaneously develop and disappear anytime during treatment with anti-TNF agents. ${ }^{36,37}$ In the study by Vande Casteele et al. ${ }^{36}$ transient ADAs were reported in $28 \%$ of patients who developed ADAs during treatment with IFX. They also observed that transient ADAs to IFX not always resulted in a worse clinical outcome, while sustained high levels of ADAs to IFX led to permanent LOR. ${ }^{36}$ In our study, we were also able to observe that $30 \%(3 / 10)$ of patients with positive ADAs to IFX had transient ADAs. Furthermore, patients with transient ADAs to IFX also had relatively lower ADA titers of borderline significance compared with those exhibiting sustained ADAs and those who had switched to ADL due to secondary LOR $(75.60 \pm 68.82 \mathrm{AU} / \mathrm{mL}$ vs $14.97 \pm 4.37 \mathrm{AU} /$ $\mathrm{mL} ; \mathrm{p}=0.059$ ). These results are in line with a recent study by Brandse et al. ${ }^{38}$ reporting that ATI titers of $30 \mathrm{AU} / \mathrm{mL}$ were consistently associated with undetectable IFX TLs.

While the presence of ADAs is responsible for the decrease of anti-TNF drug levels, recent evidence also suggests that the development of ADAs could conversely be a result of insufficient anti-TNF exposure. ${ }^{10,11}$ The probability of developing ADAs to IFX has been reported to correlate with the amount of time during which IFX TLs remain below $3 \mathrm{mg} / \mathrm{mL} .{ }^{38,39}$ To this end, we found a significant association between IFX TLs and ADA positivity in patients receiving IFX in our study and revealed that a receiver operating characteristic curve-based optimal IFX TL cutoff of $1.88 \mu \mathrm{g} / \mathrm{mL}$ was associated with ADA positivity. Proactive TDM, which pre-emptively optimizes treatment based on TDM in patients in clinical remission during maintenance therapy instead of waiting for and acting upon secondary LOR is now receiving increasing interest in the treatment of IBD. ${ }^{11,40}$ Recent randomized controlled trials involving both adults and children have revealed promising results of its superiority over clinically based dosing or reactive TDM based dosing. ${ }^{41,42}$ The currently acknowledged therapeutic threshold TLs for clinical remission in adult patients with IBD are $5 \mu \mathrm{g} / \mathrm{mL}$ for IFX, and $7.5 \mu \mathrm{g} / \mathrm{mL}$ for ADL. ${ }^{19}$ However, mucosal healing, which is the current target for the treatment for IBD, is a more stringent goal compared with clinical remission, and therefore requires higher TL thresh- olds. Although there is limited data on the TL thresholds required for mucosal healing, studies of both adult and pediatric patients with IBD have proposed higher cutoffs for mucosal healing compared with those for clinical remission. $^{43,44}$

Unfortunately, appropriate TDM is currently not available in Korea. Tests for IFX TLs have only recently been made available in clinical practice, and tests for ADAs against IFX as well as for TDM of other biologics are currently unavailable in Korea. Moreover, for dose intensification or interval shortening, the current national insurance policy of Korea reimburses only in the event of clinically apparent secondary LOR. The results of our study may therefore provide the necessity for the implementation of tests for ADAs to IFX as well as for TDM of other antiTNF agents in real-life clinical practice. Future studies based on serial and proactive TDM are required to better elucidate the relationship between IFX TLs and the development of ADAs.

Meanwhile, two patients exhibiting sustained ADA responses against IFX had continuously sustained clinical remission for a year without experiencing any change in dosing or interval changes in IFX. Considering that these patients had FC levels of $<100 \mathrm{mg} / \mathrm{kg}$ at baseline and 1-year follow-up TDM measurement, it could be assumed that both patients had mucosal healing. According to studies on anti-TNF cessation in adults and pediatric patients with $\mathrm{CD}$, both could be good candidates for anti-TNF cessation in those who would likely continue to maintain clinical remission for a long period, receiving immunomodulatory therapy only. ${ }^{45-49}$ Therefore, TDM carried out on a regular basis may also inform the identification of patients that could consider discontinuing anti-TNF agents.

The major limitation of this study is the cross-sectional study design. Only patients receiving maintenance treatment were included, while those who had discontinued anti-TNF treatment due to primary or secondary LOR were not, and hence selection bias may have been introduced. Moreover, we lacked serial test results for ADAs and TLs starting from anti-TNF initiation. It is known that $90 \%$ of patients who develop a sustained ADA response against IFX do so within the first 12 months of therapy, while transient ADAs to IFX are detected throughout the period of IFX treatment. ${ }^{50}$ Because, the blood samples for measurement of ADAs of IFX were taken at a time period of $3.0 \pm 1.3$ years from IFX initiation, the proportion of patients who had actually developed ADAs to IFX may not be accurate. Another limitation is the relatively small number of patients included. Large-scale prospective studies are required to increase our insight.

In conclusion, IFX TLs were associated with the devel- 
opment of ADAs in pediatric patients with IBD receiving maintenance treatment with IFX. Future studies based on serial and proactive TDM are required to better elucidate the relationship between IFX TLs and the development of ADAs.

\section{CONFLICTS OF INTEREST}

No potential conflict of interest relevant to this article was reported.

\section{ACKNOWLEDGEMENTS}

This work was supported by the National Research Foundation of Korea (NRF) grant funded by the Korean government (MSIT) (No. 2017R1C1B5076980).

\section{AUTHOR CONTRIBUTIONS}

Conceptualization: B.K. Data curation: J.Y.K., Y.L., B.K. Formal analysis: Y.L., B.H.C., B.K. Funding acquisition: B.K. Methodology: B.H.C., B.K. Project administration: B.K. Visualization: J.Y.K., Y.L., B.K. Writing - original draft: J.Y.K., Y.L., B.K. Writing - review \& editing: B.H.C., B.K. Approval of final manuscript: all authors.

\section{ORCID}

Ju Young Kim https://orcid.org/0000-0002-4406-2428 Yoon Lee https://orcid.org/0000-0001-9521-3575 Byung-Ho Choe https://orcid.org/0000-0001-9899-9120 Ben Kang https://orcid.org/0000-0002-8516-9803

\section{REFERENCES}

1. Baumgart DC, Sandborn WJ. Inflammatory bowel disease: clinical aspects and established and evolving therapies. Lancet 2007;369:1641-1657.

2. Rosen MJ, Dhawan A, Saeed SA. Inflammatory bowel disease in children and adolescents. JAMA Pediatr 2015;169:1053-1060.

3. Kang B, Kim JE, Jung JH, et al. Korean children and adolescents with Crohn's disease are more likely to present with perianal fistulizing disease at diagnosis compared to their European counterparts. Pediatr Gastroenterol Hepatol Nutr 2020;23:49-62.
4. Kim S. Surgery in pediatric Crohn's disease: indications, timing and post-operative management. Pediatr Gastroenterol Hepatol Nutr 2017;20:14-21.

5. Kang B, Choi SY, Kim HS, Kim K, Lee YM, Choe YH. Mucosal healing in paediatric patients with moderate-to-severe luminal Crohn's disease under combined immunosuppression: escalation versus early treatment. J Crohns Colitis 2016;10:1279-1286.

6. Choi J, Kang B, Kim MJ, Sohn I, Lee HJ, Choe YH. Early infliximab yields superior long-term effects on linear growth in pediatric Crohn's disease patients. Gut Liver 2018;12:255262.

7. Kang B, Choe YH. Early biologic treatment in pediatric Crohn's disease: catching the therapeutic window of opportunity in early disease by treat-to-target. Pediatr Gastroenterol Hepatol Nutr 2018;21:1-11.

8. Moss AC, Brinks V, Carpenter JF. Review article: immunogenicity of anti-TNF biologics in IBD: the role of patient, product and prescriber factors. Aliment Pharmacol Ther 2013;38:1188-1197.

9. Vermeire S, Gils A, Accossato P, Lula S, Marren A. Immunogenicity of biologics in inflammatory bowel disease. Therap Adv Gastroenterol 2018;11:1756283X17750355.

10. Roblin X, Riviere P, Flamant M, et al. Proactive therapeutic drug monitoring of TNF antagonists in inflammatory bowel disease. Inflamm Bowel Dis 2018;24:1904-1909.

11. Brandse JF, Mathôt RA, van der Kleij D, et al. Pharmacokinetic features and presence of antidrug antibodies associate with response to infliximab induction therapy in patients with moderate to severe ulcerative colitis. Clin Gastroenterol Hepatol 2016;14:251-258.

12. Nanda KS, Cheifetz AS, Moss AC. Impact of antibodies to infliximab on clinical outcomes and serum infliximab levels in patients with inflammatory bowel disease (IBD): a metaanalysis. Am J Gastroenterol 2013;108:40-47.

13. Colombel JF, Sandborn WJ, Reinisch W, et al. Infliximab, azathioprine, or combination therapy for Crohn's disease. $\mathrm{N}$ Engl J Med 2010;362:1383-1395.

14. Lichtenstein GR, Diamond RH, Wagner CL, et al. Clinical trial: benefits and risks of immunomodulators and maintenance infliximab for IBD-subgroup analyses across four randomized trials. Aliment Pharmacol Ther 2009;30:210-226.

15. Yarur AJ, Rubin DT. Therapeutic drug monitoring of antitumor necrosis factor agents in patients with inflammatory bowel diseases. Inflamm Bowel Dis 2015;21:1709-1718.

16. Levine A, Koletzko S, Turner D, et al. ESPGHAN revised porto criteria for the diagnosis of inflammatory bowel disease in children and adolescents. J Pediatr Gastroenterol Nutr 2014;58:795-806.

17. Kang B, Lee Y, Lee K, Choi YO, Choe YH. Long-term outcomes after switching to CT-P13 in pediatric-onset inflam- 
matory bowel disease: a single-center prospective observational study. Inflamm Bowel Dis 2018;24:607-616.

18. Choi SY, Choi YO, Choe YH, Kang B. Potential utility of therapeutic drug monitoring of adalimumab in predicting short-term mucosal healing and histologic remission in pediatric Crohn's disease patients. J Korean Med Sci 2020;35:e114.

19. Vande Casteele N, Herfarth H, Katz J, Falck-Ytter Y, Singh S. American Gastroenterological Association Institute technical review on the role of therapeutic drug monitoring in the management of inflammatory bowel diseases. Gastroenterology 2017;153:835-857.

20. Carman N, Mack DR, Benchimol EI. Therapeutic drug monitoring in pediatric inflammatory bowel disease. Curr Gastroenterol Rep 2018;20:18.

21. Sharma S, Eckert D, Hyams JS, et al. Pharmacokinetics and exposure-efficacy relationship of adalimumab in pediatric patients with moderate to severe Crohn's disease: results from a randomized, multicenter, phase- 3 study. Inflamm Bowel Dis 2015;21:783-792.

22. Matar M, Shamir R, Turner D, et al. Combination therapy of adalimumab with an immunomodulator is not more effective than adalimumab monotherapy in children with Crohn's disease: a post hoc analysis of the PAILOT randomized controlled trial. Inflamm Bowel Dis 2020;26:1627-1635.

23. Ungar B, Engel T, Yablecovitch D, et al. Prospective observational evaluation of time-dependency of adalimumab immunogenicity and drug concentrations: the POETIC study. Am J Gastroenterol 2018;113:890-898.

24. Ordás I, Mould DR, Feagan BG, Sandborn WJ. Anti-TNF monoclonal antibodies in inflammatory bowel disease: pharmacokinetics-based dosing paradigms. Clin Pharmacol Ther 2012;91:635-646.

25. Vande Casteele N, Khanna R, Levesque BG, et al. The relationship between infliximab concentrations, antibodies to infliximab and disease activity in Crohn's disease. Gut 2015;64:1539-1545.

26. Ben-Horin S, Chowers Y. Review article: loss of response to anti-TNF treatments in Crohn's disease. Aliment Pharmacol Ther 2011;33:987-995.

27. Choi SY, Kang B, Lee JH, Choe YH. Clinical use of measuring trough levels and antibodies against infliximab in patients with pediatric inflammatory bowel disease. Gut Liver 2017;11:55-61.

28. Merras-Salmio L, Kolho KL. Clinical use of infliximab trough levels and antibodies to infliximab in pediatric patients with inflammatory bowel disease. J Pediatr Gastroenterol Nutr 2017;64:272-278.

29. O’Meara S, Nanda KS, Moss AC. Antibodies to infliximab and risk of infusion reactions in patients with inflammatory bowel disease: a systematic review and meta-analysis. In- flamm Bowel Dis 2014;20:1-6.

30. Vermeire S, Noman M, Van Assche G, Baert F, D’Haens G, Rutgeerts P. Effectiveness of concomitant immunosuppressive therapy in suppressing the formation of antibodies to infliximab in Crohn's disease. Gut 2007;56:1226-1231.

31. Kansen HM, van Rheenen PF, Houwen RHJ, et al. Less antiinfliximab antibody formation in paediatric Crohn patients on concomitant immunomodulators. J Pediatr Gastroenterol Nutr 2017;65:425-429.

32. Chi LY, Zitomersky NL, Liu E, et al. The impact of combination therapy on infliximab levels and antibodies in children and young adults with inflammatory bowel disease. Inflamm Bowel Dis 2018;24:1344-1351.

33. Qiu Y, Mao R, Chen BL, et al. Effects of combination therapy with immunomodulators on trough levels and antibodies against tumor necrosis factor antagonists in patients with inflammatory bowel disease: a meta-analysis. Clin Gastroenterol Hepatol 2017;15:1359-1372.

34. Ma C, Battat R, Jairath V, Vande Casteele N. Advances in therapeutic drug monitoring for small-molecule and biologic therapies in inflammatory bowel disease. Curr Treat Options Gastroenterol 2019;17:127-145.

35. Feuerstein JD, Nguyen GC, Kupfer SS, Falck-Ytter Y, Singh S; American Gastroenterological Association Institute Clinical Guidelines Committee. American Gastroenterological Association Institute guideline on therapeutic drug monitoring in inflammatory bowel disease. Gastroenterology 2017;153:827-834.

36. Vande Casteele N, Gils A, Singh S, et al. Antibody response to infliximab and its impact on pharmacokinetics can be transient. Am J Gastroenterol 2013;108:962-971.

37. Yanai H, Lichtenstein L, Assa A, et al. Levels of drug and antidrug antibodies are associated with outcome of interventions after loss of response to infliximab or adalimumab. Clin Gastroenterol Hepatol 2015; 13:522-530.

38. Brandse JF, Mould D, Smeekes O, et al. A real-life population pharmacokinetic study reveals factors associated with clearance and immunogenicity of infliximab in inflammatory bowel disease. Inflamm Bowel Dis 2017;23:650-660.

39. Lega S, Bramuzzo M, Dubinsky MC. Therapeutic drug monitoring in pediatric IBD: current application and future perspectives. Curr Med Chem 2018;25:2840-2854.

40. Ben-Horin S. Drug level-based anti-tumor necrosis factor therapy: ready for prime time? Gastroenterology 2015;148:1268-1271.

41. Vande Casteele N, Ferrante M, Van Assche G, et al. Trough concentrations of infliximab guide dosing for patients with inflammatory bowel disease. Gastroenterology 2015;148:1320-1329.

42. Assa A, Matar M, Turner D, et al. Proactive monitoring of adalimumab trough concentration associated with increased 
clinical remission in children with Crohn's disease compared with reactive monitoring. Gastroenterology 2019;157:985996.

43. Ungar B, Levy I, Yavne Y, et al. Optimizing anti-TNF- $a$ therapy: serum levels of infliximab and adalimumab are associated with mucosal healing in patients with inflammatory bowel diseases. Clin Gastroenterol Hepatol 2016;14:550-557.

44. Kang B, Choi SY, Choi YO, et al. Infliximab trough levels are associated with mucosal healing during maintenance treatment with infliximab in paediatric Crohn's disease. J Crohns Colitis 2019;13:189-197.

45. Louis E, Mary JY, Vernier-Massouille G, et al. Maintenance of remission among patients with Crohn's disease on antimetabolite therapy after infliximab therapy is stopped. Gastroenterology 2012;142:63-70.

46. Papamichael K, Vande Casteele N, Gils A, et al. Long-term outcome of patients with Crohn's disease who discontinued infliximab therapy upon clinical remission. Clin Gastroenterol Hepatol 2015;13:1103-1110.
47. Ben-Horin S, Chowers Y, Ungar B, et al. Undetectable antiTNF drug levels in patients with long-term remission predict successful drug withdrawal. Aliment Pharmacol Ther 2015;42:356-364.

48. Torres J, Boyapati RK, Kennedy NA, Louis E, Colombel JF, Satsangi J. Systematic review of effects of withdrawal of immunomodulators or biologic agents from patients with inflammatory bowel disease. Gastroenterology 2015;149:17161730.

49. Kang B, Choi SY, Choi YO, et al. Subtherapeutic infliximab trough levels and complete mucosal healing are associated with sustained clinical remission after infliximab cessation in paediatric-onset Crohn's disease patients treated with combined immunosuppressive therapy. J Crohns Colitis 2018;12:644-652.

50. Ungar B, Chowers Y, Yavzori M, et al. The temporal evolution of antidrug antibodies in patients with inflammatory bowel disease treated with infliximab. Gut 2014;63:12581264. 